\section{New security feature for patient management software}

Dentally has now added a new feature to the two factor authentication option within the software, whereby you can download an app to your phone which will give the user a unique one off code to access Dentally - an important update for team members who may be working remotely and away from the dental practice.

Previously two factor authentication was achieved through the receipt of an SMS, but this new extra feature heightens security and means a user no longer has to wait to receive an SMS on their mobile phone. To use this new feature, dental practitioners (and their staff) simply download an authenticator app such as Authy or Google Authenticator, open it for Dentally and it will give a numerical code that the user enters into Dentally in order to complete the login process.

If you would like more information on this or other ways Dentally are working with customers to help them during these challenging times as our society works to prevent the spread of COVID-19, then email hello@dentally.co.

\title{
Flowable bioactive composite for durable restorations
}

Whether you wish to build up occlusal surfaces and anatomical details, or line cavity floors and fill occlusal or cervical cavities: the newly developed Beautifil

Flow Plus X offers the optimal flowability and dimensional stability for all indications, since it is available in two viscosities: F00

(Zero Flow) and F03 (Low Flow). This injectable

hybrid composite for anterior and posterior restorations builds on the clinically proven handling benefits of Beautifil Flow Plus; it is non-slumping, self-levelling and nondripping. Besides, SHOFU's improved Giomer Technology with nano S-PRG filler particles enhances polishability, gloss retention and bioactivity - for sustainable, long-term protection against recurrent caries.

The composite is available in nine common tooth shades plus various dentine, enamel, bleach and effect shades, based on the shade range and featuring the proven shade stability of the entire Beautifil product line.

For further information contact Shofu - 01732 783580, sales@shofu.co.uk, www. shofu.co.uk.

\section{Step into the future, today}

Essential equipment maintenance can be a real stress and strain on your time in practice. So why not step into the future of maintenance by choosing Total Air Care from DentalAir?

A comprehensive approach that covers all of your dental air compressor needs, signing up to this package is priced from as little as $£ 14.95$ per week and includes the installation of a new, oil-free dental air compressor. With Total Air Care you can rest assured that your dental air compressor will remain compliant and provide sterile air for use throughout

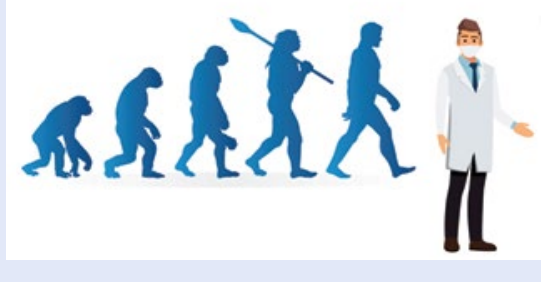

your practice. Plus, should anything ever go wrong, you know it will be up and running again in no time thanks to our swift, national breakdown service.

For more information, contact

DentalAir at info@dentalair.com or call 08009757530 .

\section{The ideal composites for wear cases}

Venus Pearl and Venus Diamond are sister composites with full-mouth indication. They are powered by a unique state-of-the-art TCD resin. The TCD ingredient, developed by the Kulzer scientists, helps to provide significantly higher mechanical properties which results in less chipping and material failure.

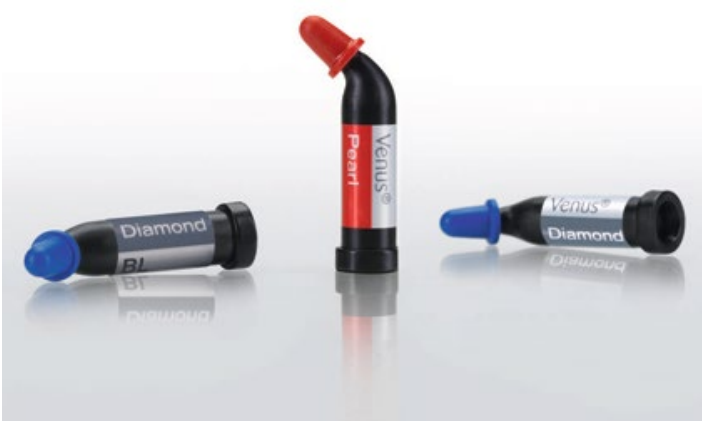

TCD is a special ingredient that increases the degree of conversion. Within the first 24 hours after placement, light-cured composites typically only reach 55-65\% conversion. However, Venus Pearl and Venus Diamond, powered by TCD, demonstrate a significantly higher degree of conversion to around $80 \%$. This is very similar to indirect laboratory composites. This reassuringly high conversion rate leads to less chipping over time, making Pearl and Diamond the ideal composites for wear cases, as well as incisaledge bonding where strength in thin-section is critical.

The notable difference between Pearl and Diamond is the consistency of material when being handled. Venus Pearl has a creamy feel. Venus Diamond is slightly firmer. Both materials stay precisely where they are placed, allowing the clinician to carve anatomy without fear of slumping or pullback. Furthermore, with extended workingtime under normal lighting conditions, the clinician can take their time without fear of the material setting prematurely.

Most dental composites utilise the traditional bis-gma resin that was developed back in the 1960s. However, an increasing number of patients have concern over their potential exposure to Bisphenol-A. To put their mind at rest, Venus Pearl and Venus Diamond are completely free from BPA.

https://tinyurl.com/wohxpsw 